\title{
Science et politique
}

Le message de pierre de la Bibliothèque nationale et universitaire de Strasbourg

\section{Christophe Didier}

\section{(2) OpenEdition \\ 1 Journals}

Édition électronique

URL : https://journals.openedition.org/rbnu/3088

DOI : $10.4000 /$ rbnu.3088

ISSN : 2679-6104

Éditeur

Bibliothèque nationale et universitaire de Strasbourg

\section{Édition imprimée}

Date de publication : 1 mai 2010

Pagination : 8-15

ISSN : 2109-2761

\section{Référence électronique}

Christophe Didier, «Science et politique », La Revue de la BNU [En ligne], 1 | 2010, mis en ligne le 01 janvier 2021, consulté le 13 juin 2021. URL : http://journals.openedition.org/rbnu/3088 ; DOI : https:// doi.org/10.4000/rbnu.3088

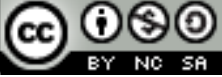

La Revue de la BNU est mise à disposition selon les termes de la Licence Creative Commons Attribution - Pas d'Utilisation Commerciale - Partage dans les Mêmes Conditions 4.0 International. 


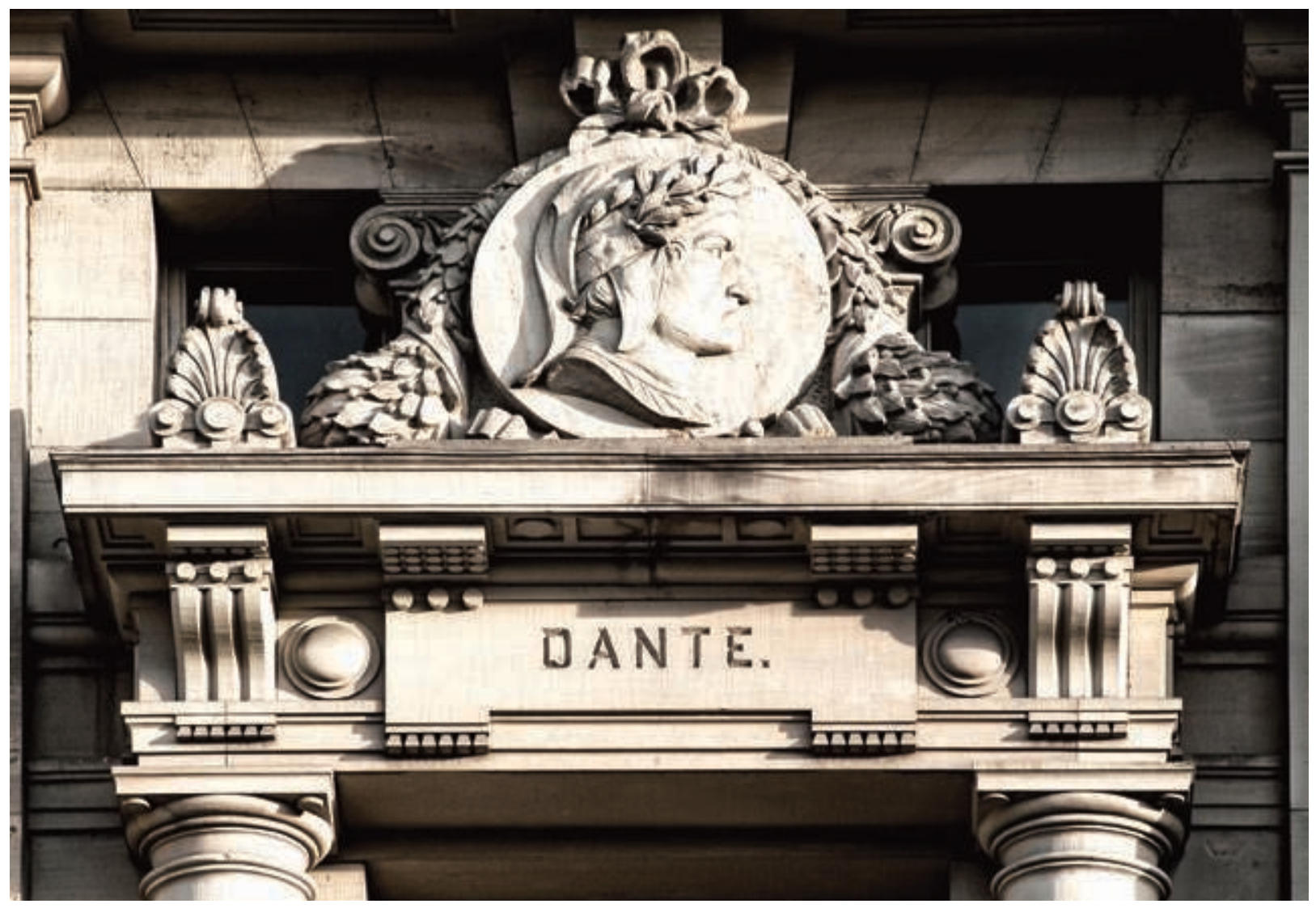

\Médaillon représentant Dante (façade ouest)

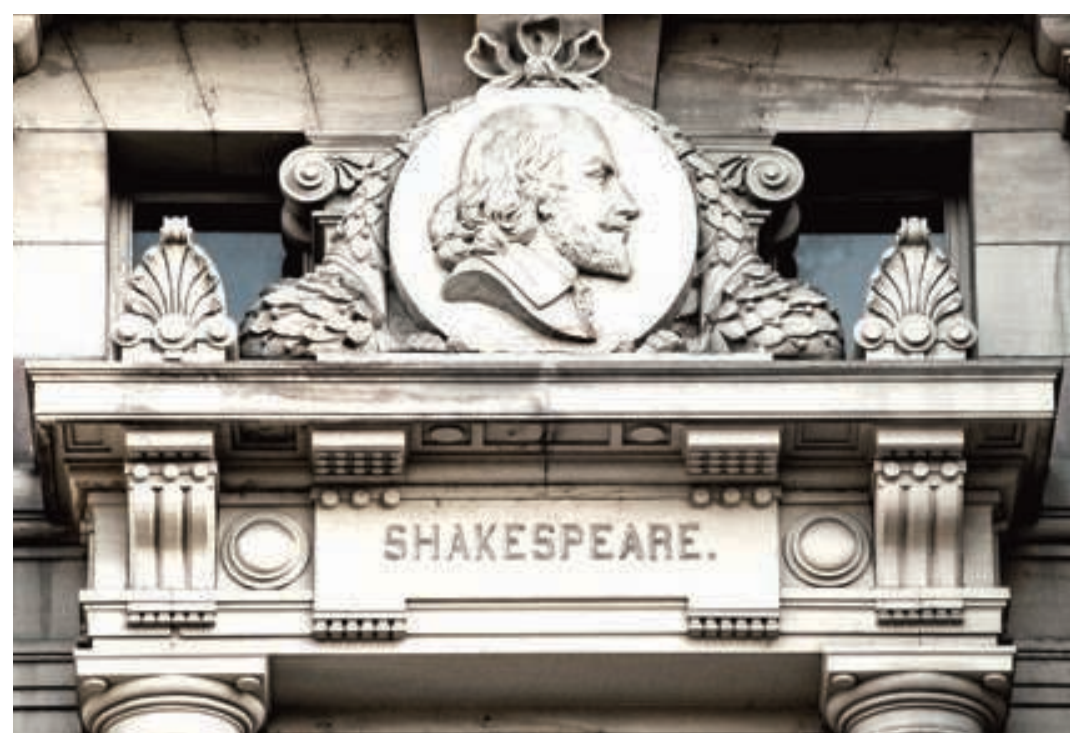

\édaillon représentant Shakespeare (façade ouest) 


\section{Science et politique : le message de pierre de la Bibliothèque nationale et universitaire de Strasbourg}

A

la fin du XIX ${ }^{\mathrm{e}}$ siècle, le nouveau bâtiment de la Bibliothèque impériale de l'université et de la région (Kaiserliche Universitätsund Landesbibliothek, l'ancêtre de la BNU) est construit dans le cœur symbolique du nouveau quartier édifié par les autorités allemandes, sur un côté de la place de l'Empereur (Kaiserplatz, actuelle place de la République). Il avoisine le parlement régional, le palais où réside l'Empereur lorsqu'il se déplace à Strasbourg et deux bâtiments abritant, suivant le système politique allemand, des ministères régionaux. Cette situation centrale donne évidemment à l'établissement culturel et éducatif qu'est la bibliothèque une fonction politique qui prend sa place dans le programme de reconquête idéologique de l'Alsace-Lorraine par le nouveau pouvoir allemand.

L'importance symbolique que revêt la construction de la bibliothèque se voit dès le début de l'histoire du bâtiment : les décisions qui la concernent (incluant des aspects techniques comme le choix des entreprises ou celui du mobilier) font ainsi l'objet de discussions régulières au parlement régional. Bien plus, la construction du nouveau bâtiment destiné à abriter ce dernier se fait de façon parallèle à celle de la bibliothèque, de sorte qu'on peut presque les considérer comme des "bâtiments jumeaux ", pour reprendre les termes de l'historien de l'architecture Klaus Nohlen : même situation sur le côté est de la place, même période de construction (de 1889 à 1895 pour la bibliothèque, de 1888 à 1892 pour le parlement), mêmes architectes (Hartel et Neckelmann), mêmes matériaux employés (grès vosgien pour l'extérieur, grès de la Forêt-Noire pour les es- caliers intérieurs), enfin même sculpteur (Johannes Riegger, Badois établi à Strasbourg) responsable de l'exécution du programme iconographique.

Car tous ces bâtiments devaient aussi être "parlants ", et furent dotés de représentations imagées, allégoriques ou historiques, qui devaient en souligner la fonction et l'inscription dans le nouvel ordre politique et culturel européen. La bibliothèque, avec des bas-reliefs, des statues et des médaillons (ainsi qu'un décor intérieur peint, malheureusement détruit dans les années cinquante) a été l'objet d'attentions particulières, mais n'est aucunement une exception, tous les bâtiments cités plus haut ayant été pareillement décorés : le parlement régional par des médaillons figurant les armes des villes d'AlsaceLorraine, le palais de l'Empereur par des cartouches représentant les armes des principales villes et familles princières d'Allemagne (en incorporant Strasbourg, Metz, l'Alsace et la Lorraine à ce programme), les bâtiments ministériels fermant la place vers le nord par des reliefs et des statues rappelant pareillement leur fonction. Enfin, un peu plus loin, l'université, la poste et l'Aubette (reconstruite comme conservatoire après l'incendie de 1870) reçurent elles aussi leur programme sculpté.

Celui de la bibliothèque est particulièrement riche et intéressant. Au fronton de la façade ouest, dominant l'entrée principale, deux groupes de statues, symbolisant les arts et les sciences, encadrent un pignon triangulaire contenant les représentations allégoriques de l'imprimerie, de la gravure sur bois, de la gravure sur cuivre, de la lithographie et de la photographie (soit toutes les disciplines convoquées pour la réalisation d'un livre). Si l'on en croit l'histo- 


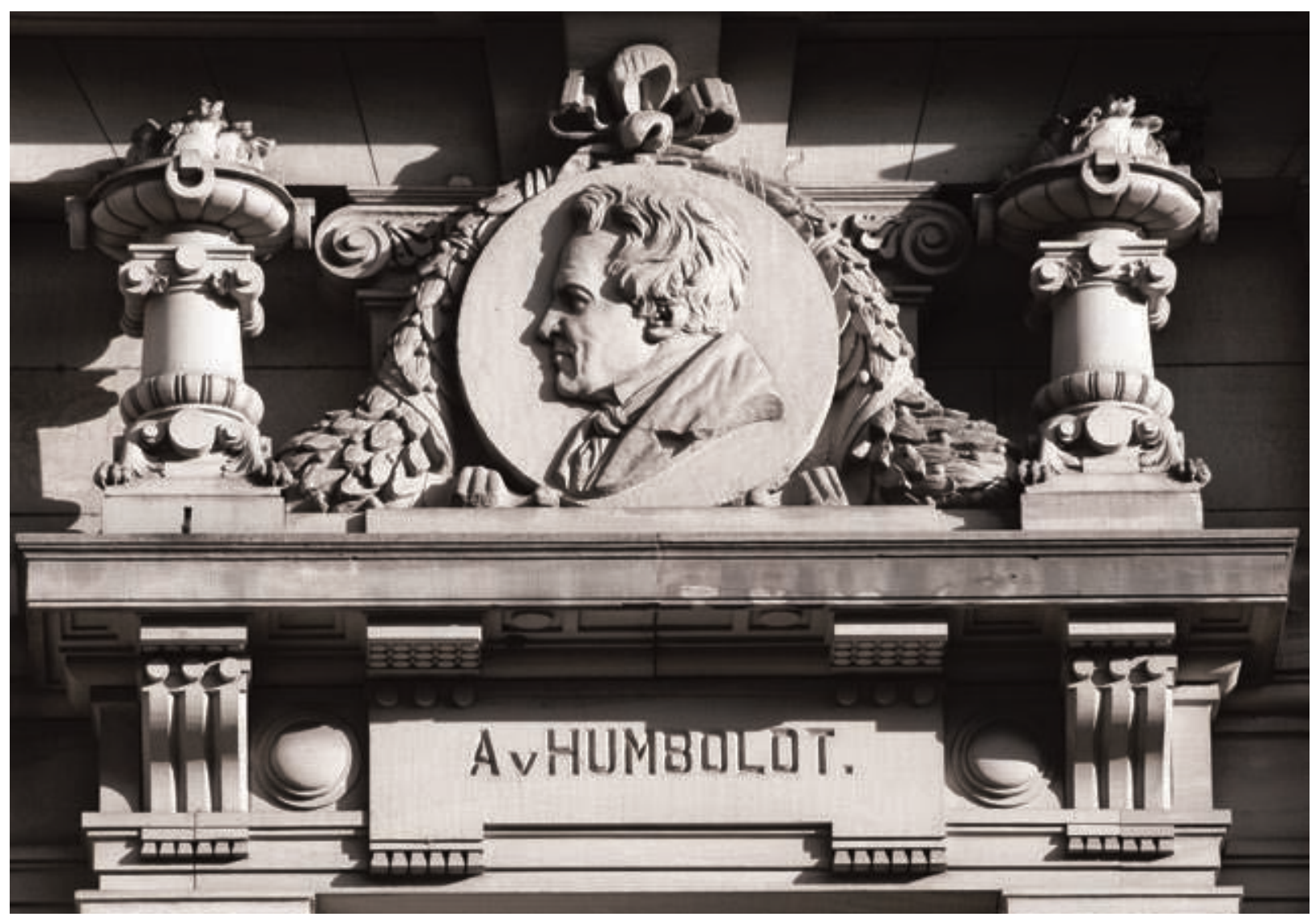

\ Médaillon représentant Humboldt (façade sud, en haut)

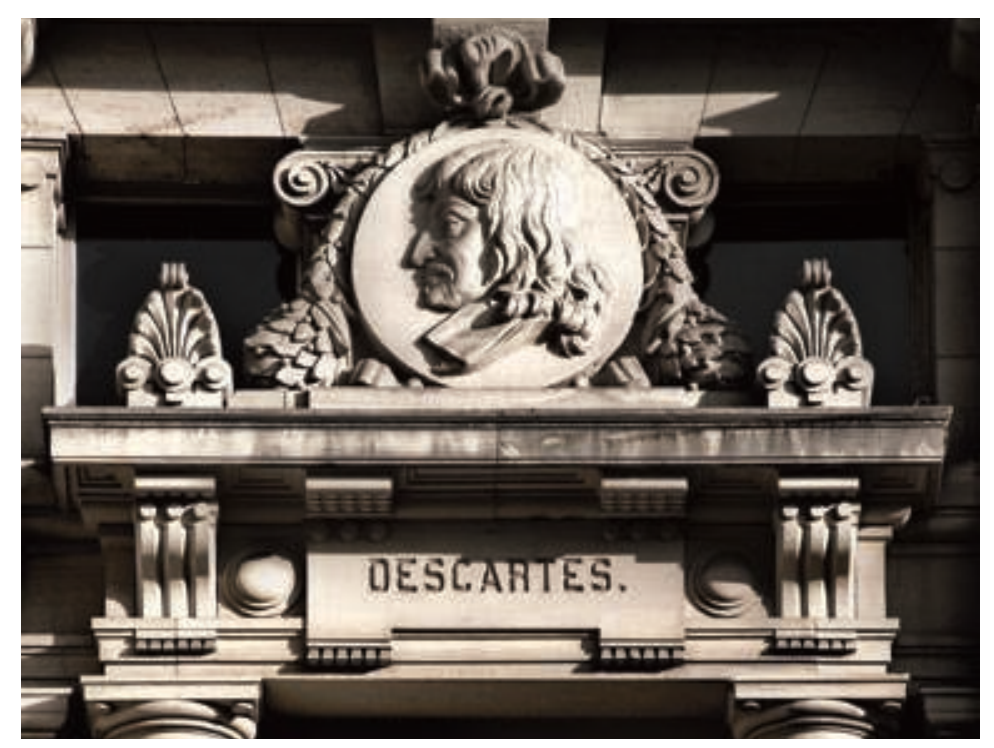

\Médaillon représentant Descartes (façade sud, en haut) 
rien Hanns Michael Crass, ce serait la première fois que la photographie serait ainsi représentée allégoriquement dans un bâtiment de bibliothèque. Sur les côtés nord et sud, deux autres pignons montrent pareillement des représentations allégoriques, les sciences et littératures de l'Antiquité au nord, celles du Moyen Âge et des Temps modernes au sud. Cet hommage à l'esprit humain est encore renforcé par le choix des éléments purement décoratifs figurés par les acrotères, des Minerves et un sphinx trônant à l'ouest, des chouettes au nord et au sud. Témoins du rôle de l'Antiquité comme socle de la connaissance actuelle, des constructions représentant des autels antiques marquent les coins du bâtiment, sur la balustrade de pierre qui précède visuellement le dôme.

Tous ces décors sculptés, placés bien en évidence à la partie avant du bâtiment et en hauteur, sont bien sûr essentiels à sa compréhension générale. Mais la partie la plus remarquable, parce que réellement programmatique, de l'ensemble iconographique consiste dans le cycle de trente-et-un médaillons qui courent tout autour du bâtiment et figurent en grande majorité des écrivains et penseurs de l'Europe (seuls les trois médaillons centraux de la façade ouest, situés sous le pignon décrit plus haut, reprennent les allégories des arts, des sciences et de leur figure antique tutélaire, le dieu Apollon).

Ce livre d'images, incarnation dans la pierre du savoir que renferme la bibliothèque, a été lui-même savamment pensé. Sur la façade principale, de part et d'autre du pignon central, quatre représentants de la littérature allemande (Gottfried von Strassburg, Lessing, Goethe et Schiller) et quatre représentants des littératures européennes (Molière, Calderón, Dante, Shakespeare) ont tous le regard tourné vers les médaillons centraux d'Apollon, des sciences et des arts. Sur les côtés nord et sud, mais encore dans la partie avant du bâtiment, on trouve quatre repré- sentants de la littérature et de la philosophie antiques (Cicéron, Virgile, Aristote et Homère) et quatre des sciences et de la philosophie modernes (Alexander von Humboldt, Descartes, Leibnitz et Kepler), répondant, dans les deux cas, aux représentations allégoriques des pignons correspondants. Les personnages de ces huit médaillons ont le regard porté vers l'avant du bâtiment.

La partie arrière n'est pas moins bien pourvue, symboliquement là encore, puisque correspondant aux magasins à livres. Sur les côtés et en hauteur, trois groupes de deux personnages symbolisent les facultés de l'université de Strasbourg : au sud Hippocrate et Grotius pour la médecine et le droit, au nord Erasme et Scaliger pour la philosophie et la philologie, à l'est, sur l'arrière, Thomas d'Aquin pour la théologie catholique et Melanchton pour la protestante. Endessous de ces six personnages, mais placés au même endroit, six médaillons célèbrent des grands noms de l'Alsace-Lorraine : sous Thomas d'Aquin et Melanchton prennent place Geiler et Bucer ; sur les côtés sont figurés trois représentants de chacun des "Bezirke " (arrondissements) de l'Alsace-Lorraine d'alors : Jean Sturm pour la Basse-Alsace, Dom Calmet pour la Lorraine, Martin Schongauer pour la Haute-Alsace. Le dernier médaillon est symboliquement consacré à Herrade de Landsberg, faisant ainsi écho, par le biais de la tragédie de l'Hortus deliciarum '1, à l'ancienne bibliothèque et traçant ainsi un pont entre les deux institutions. Chaque groupe de deux personnages a les yeux tournés l'un vers l'autre, évitant ainsi les regards vers l'arrière et suggérant le dialogue entre les divers savoirs et les diverses régions du Reich. Afin de rendre les choses parfaitement explicites, les noms des célébrités sont gravés en toutes lettres sous les médaillons, complétés pour les locaux (dont on craignait sans doute qu'ils ne soient guère connus) par leurs dates de naissance et de mort. 
Les visées de ce programme iconographique sont assez claires : les figures représentant les différents savoirs de l'Antiquité et des Temps modernes appartiennent, grâce à l'imprimerie et aux autres techniques de reproduction figurées sur le pignon principal, aux témoignages de l'art et de la science conservés dans l'établissement. La bibliothèque se veut non seulement scientifiquement encyclopédique, mais plus encore universelle dans ses fondements, en n'oubliant aucune époque de l'Antiquité aux Temps modernes et en laissant sa place à toutes les grandes cultures européennes. La forte présence d' " étrangers ", combinée à celle de gloires locales, doit ainsi donner l'impression d'un établissement bien ancré dans sa région, mais en même temps ouvert sur le monde. Naturellement, la présence germanique est forte, $\mathrm{y}$ compris dans les allusions locales appartenant pour la plupart aux $\mathrm{XV}^{\mathrm{e}}$ et $\mathrm{XVI}^{\mathrm{e}}$ siècles, époque où le Saint-Empire gardait encore sa vocation unificatrice et correspondant à un incontestable âge d'or artistique et culturel des pays germaniques. A une époque où un nouvel Empire allemand venait d'être créé, ces allusions n'étaient évidemment pas gratuites. Mais dans l'ensemble, la visée propagandiste de cet établissement culturel apparaît plus complexe que celle d'autres bâtiments plus administratifs et utilitaires (comme la gare, la poste ou le parlement régional) dont le programme iconographique devait davantage symboliser la prise de pouvoir de l'autorité allemande dans les nouvelles provinces. Klaus Nohlen, déjà cité, rappelle que l'identité visuelle de tous les bâtiments ainsi décorés était au service d'une légitimation nationale : il fallait fédérer les deux régions du Reichsland Elsass-Lothringen, dont l'histoire jusque-là n'avait guère été commune, afin de créer une véritable conscience régionale. C'est le message que délivrent, par exemple, les figures liées de l'Alsace et de la Moselle sur la façade de la gare, ou encore, sur le palais de l'Empereur, l'héraldique du Reichsland intégrée à celle du Reich dans son ensemble. Parallèlement, il fallait suggérer la continuité de ce dernier du Saint-Empire aux temps actuels, et donc l'appartenance " naturelle " de l'Alsace-Lorraine à cet ensemble : c'était le rôle des deux fresques du hall d'entrée de la gare (aujourd'hui disparues), intitulées respectivement « Dans l'ancien Empire " et " Dans le nouvel Empire "; c'était aussi, sur la façade de la poste centrale, celui des deux groupes de statues

(elles aussi disparues) représentant la filiation du Moyen Âge (Barberousse, Maximilien $\mathrm{I}^{\text {er }}$ et Rudolf von Habsburg) aux Temps modernes (Guillaume I ${ }^{\text {er }}$, Frédéric III et Guillaume II).

A la bibliothèque par contre, la décoration se rapporte de façon moins directe à la politique vis-àvis de l'Alsace-Lorraine, et peut davantage se lire comme la preuve de l'ouverture au monde de la science allemande, à une époque où celle-ci jouait d'ailleurs un rôle phare dans bien des disciplines. Le spectre européen qui se déploie sur les murs doit témoigner de ce caractère ouvert ainsi que du rôle de l'esprit allemand pour l'Europe. Il vient en complément de la décoration sculptée de l'université, dont les trente-deux statues de savants, très majoritairement allemands cette foisci, peuvent être comprises comme la volonté d'affirmer l'excellence de l'enseignement local et le fait que l'université de Strasbourg est tout autant l'héritière de la science et de la pensée allemandes que n’importe quel autre établissement du Reich.

L'identité visuelle de la Kaiserliche Universitätsund Landesbibliothek est donc porteuse d'une visée propagandiste, et le message que diffusent ses médaillons, même atténué par rapport à celui d'autres constructions contemporaines, ne peut être compris si l'on fait abstraction du contexte politique de l'épo- 


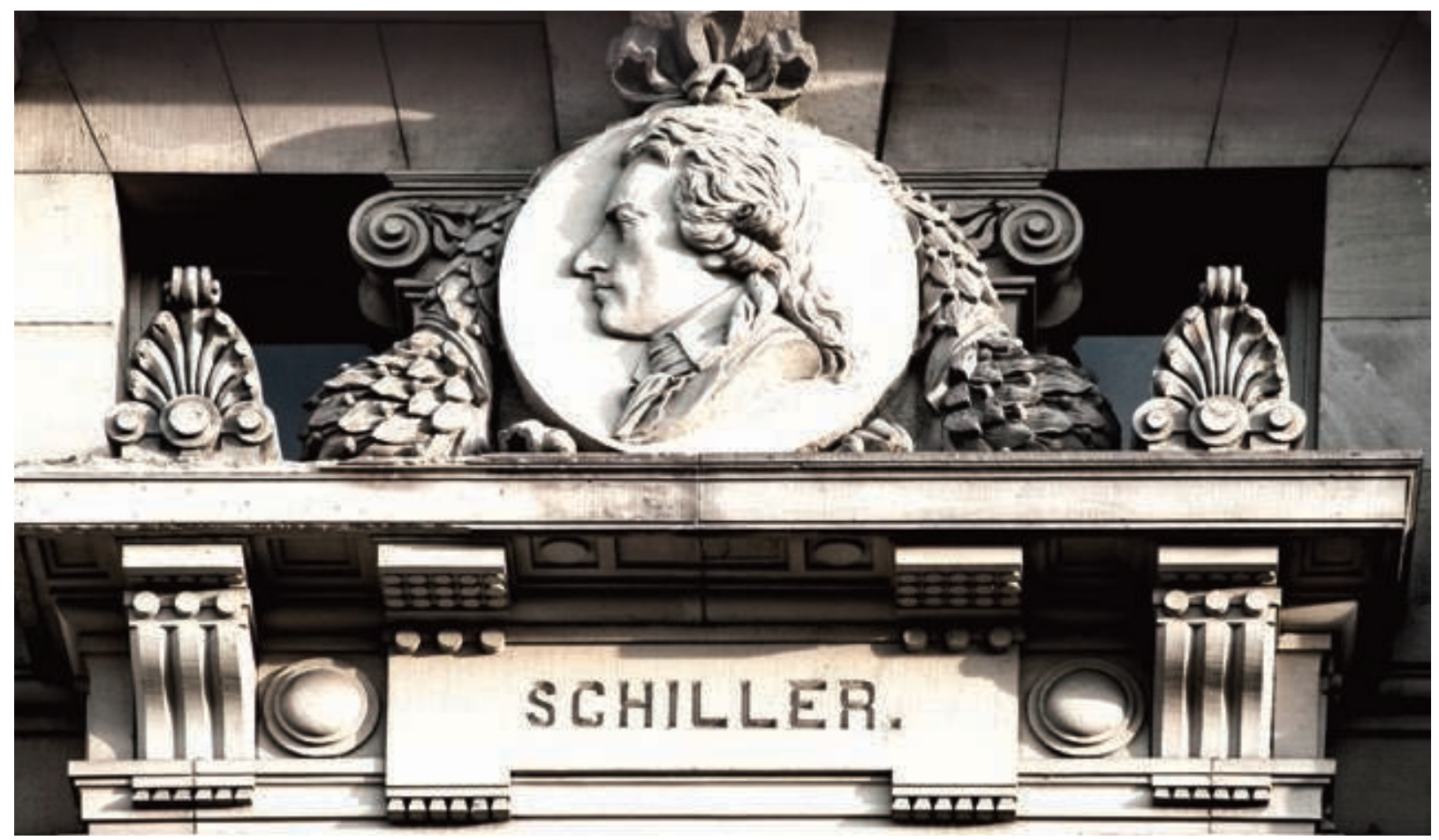

\ Médaillon représentant Schiller (façade ouest)

que. Lors de son discours inaugural de 1895 , le recteur de l'université parlait d'ailleurs de la bibliothèque comme d'un " bastion de la science allemande " (" eine Hochburg deutscher Wissenschaft"). Mais l'iconographie est dans ce cas bien plus qu'un simple miroir de la propagande. Car à la même époque se constituaient à l'ombre des médaillons des collections qui en étaient (et sont encore) le reflet parfait, dans leur visée internationale, pluridisciplinaire et ouverte sur toutes les époques. L'adéquation entre le message délivré sur les murs et l'activité réelle de l'établissement était tout aussi manifeste que la fonction politique de ce dernier sur la Kaiserplatz, et de ce fait mérite d'être soulignée. Elle le fut d'ailleurs, dès le retour de l'Alsace à la France après 1918, par un des moins germanophiles parmi les intellectuels français, Maurice Barrès en l'occurrence, qui constatait « qu'avec ses douze cent mille volumes et le soin particulier qui fut donné à l'accroissement de certaines de ses parties, la bibliothèque universitaire et régionale se place immédiatement après notre bibliothèque nationale. "
ORIENTATIONS

BIBLIOGRAPHIQUES :

Klaus NOHLEN / Baupolitik im Reichsland Elsass-Lothringen 1871-1918. - Berlin : Mann, 1982

Sebastian HAUSMANN / Die Kaiserliche Universitäts- und Landesbibliothek in Strassburg. Strasbourg : Verlag von Karl J. Trübner, 1895

Strassburg und seine Bauten / Strasbourg :

Verlag von Karl J. Trübner, 1894

Hanns Michael CRASS / Bibliotheksbauten des 19. Jahrhunderts in Deutschland. - München : Verlag Dokumentation, 1976

Maurice BARRÈs / Au retour de Strasbourg, in L’Echo de Paris, 6 décembre 1920

\section{Note}

1 - Manuscrit enluminé réalisé sous la direction de cette abbesse, un des fleurons de l'ancienne bibliothèque de la Ville de Strasbourg, brûlé avec toute la collection dans l'incendie du 25 août 1870 qui fit suite au siège de Strasbourg par les troupes prussiennes.

\section{Christophe Didier}




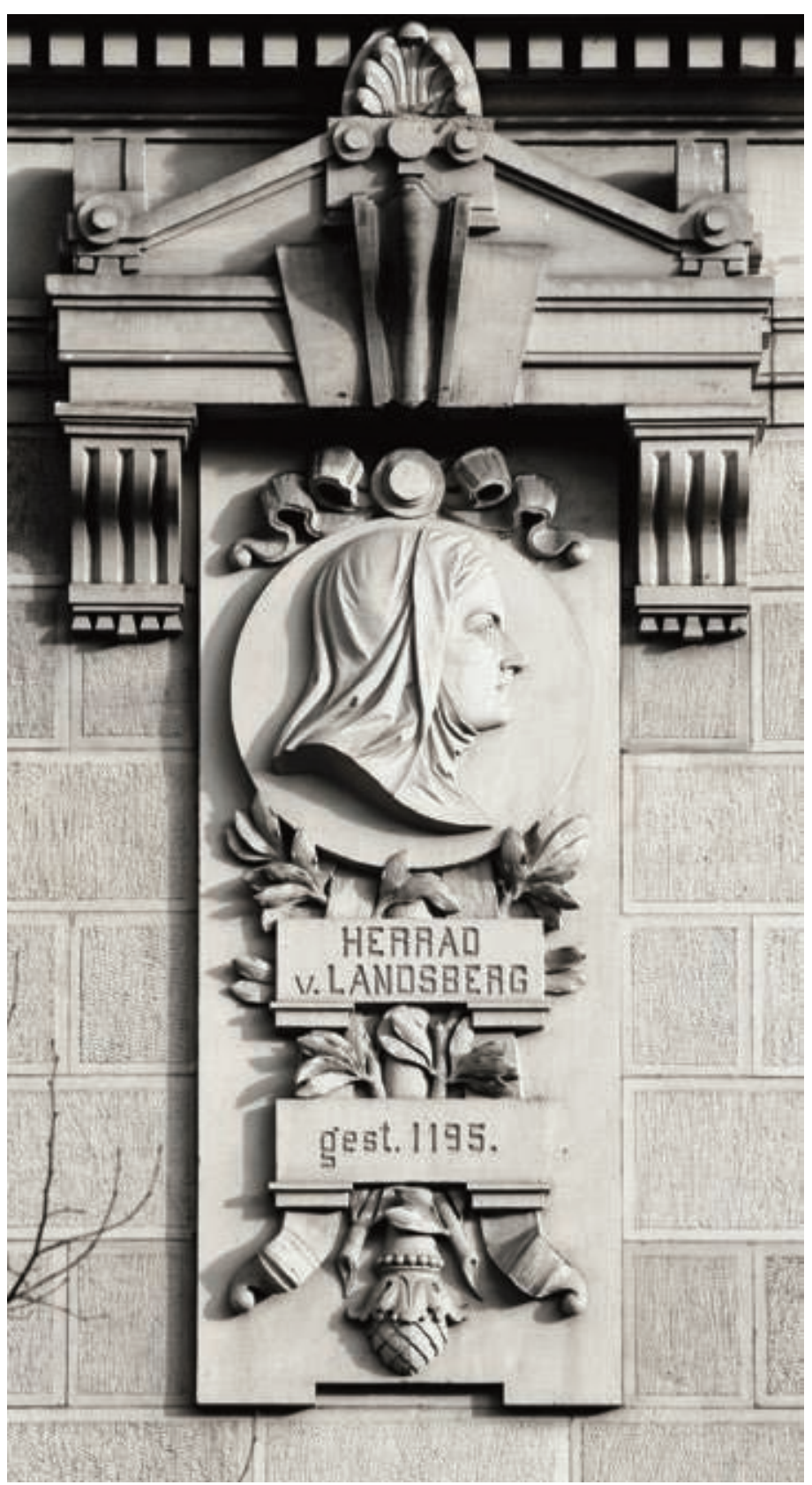

\ Médaillon représentant Herrade de Landsberg (façade sud, en bas), allusion symbolique à l'ancienne bibliothèque de la ville. 


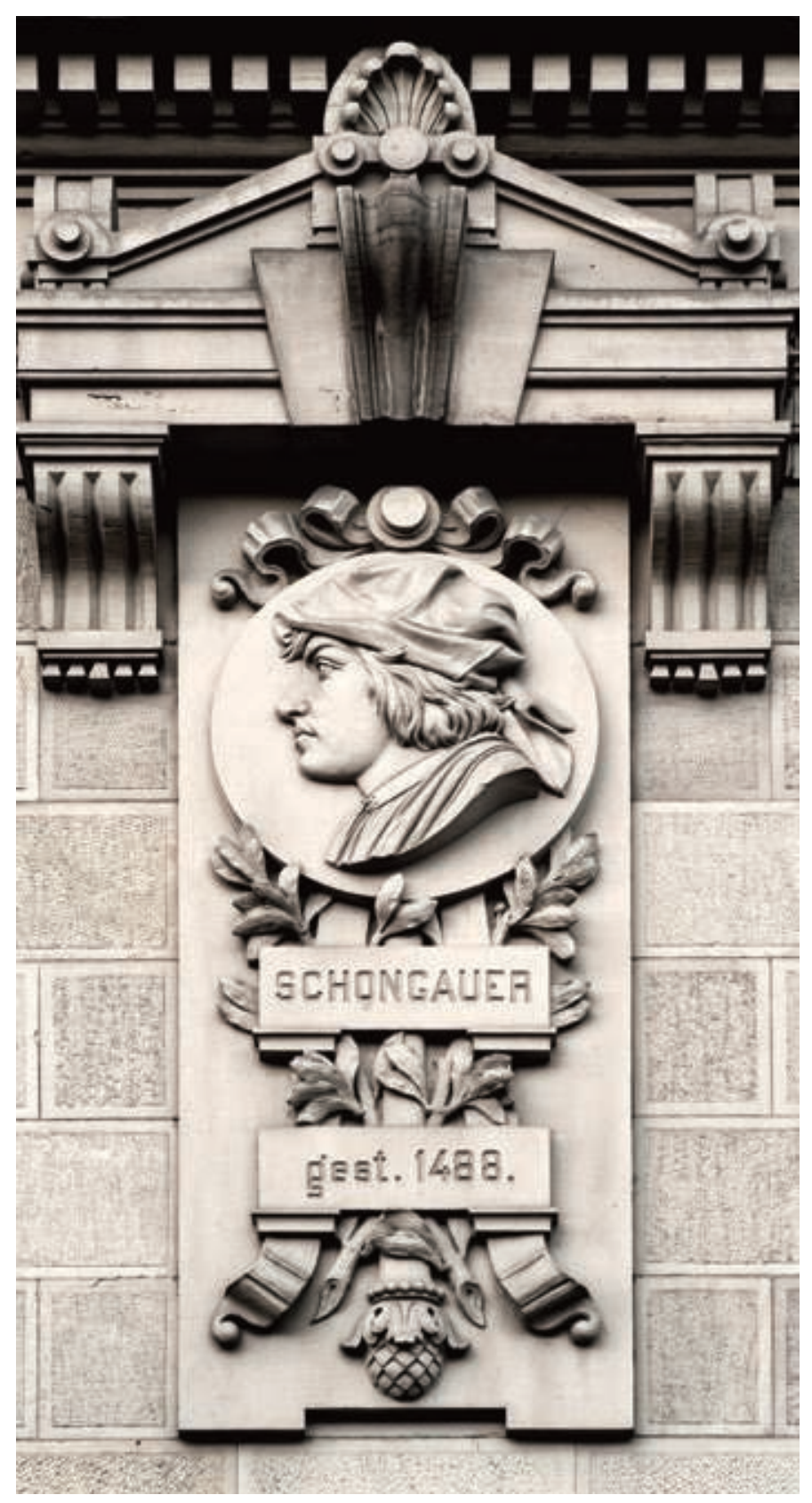

\Médaillon représentant Schongauer (façade sud, en bas).

Dans le programme iconographique, Schongauer représente

la Haute-Alsace. 